\title{
Onset of nephrotic syndrome concomitant to SARS-CoV-2 infection in a 3-year-old child
}

\author{
Alessia Morreale $^{1} \cdot$ Maria Luisa Casciana $^{1}$
}

Received: 10 August 2021 / Revised: 10 August 2021 / Accepted: 11 August 2021 / Published online: 14 October 2021

(c) The Author(s), under exclusive licence to International Pediatric Nephrology Association 2021

Dear Editors,

We read with interest the brief report from Alvarado et al. [1] describing the first pediatric case of nephrotic syndrome onset concomitant to SARS-CoV-2 infection.

A child aged 3 years and 4 months was treated in our Pediatric Unit for nephrotic syndrome onset concomitant to SARS-CoV-2 infection. Unlike the case previously described [1], our patient did not present respiratory involvement and no treatment for SARS-CoV-2 was administered.

The patient was admitted to our Emergency Department (ED) in April 2021 for abdominal distension and edema in the lower limbs in the previous 3 days. Past medical history was not significant. The child was born at term from Italian nonconsanguineous parents. Blood pressure resulted high for age $(119 / 80 \mathrm{mmHg})$. Weight was $16 \mathrm{~kg}$. Urine analysis showed a significant proteinuria ( $>400 \mathrm{mg} / \mathrm{dl}$ ). Ten days before ED admission, the patient had close contact with a COVID-19-positive family member. Molecular test for SARS-CoV-2 resulted positive in the patient and he was hospitalized in the COVID pediatric ward of our hospital. Blood exams showed hypoalbuminemia (16 g/l), hypocalcemia $(8 \mathrm{mg} / \mathrm{dl})$, increased fibrinogen $(865 \mathrm{mg} / \mathrm{dl})$, antithrombin III reduction (50\%), increased LDL cholesterol (273 mg/dl), and hypogammaglobulinemia (IgG $91 \mathrm{mg} / \mathrm{dl}$ ). C-reactive protein (CRP) resulted negative. Autoimmune tests (C3, C4, ANA, ENA) and viral serologies (HIV, $\mathrm{HBV}, \mathrm{HCV}$ ) were negative. Clinical features and laboratory results were consistent with onset of nephrotic syndrome and treatment with oral steroids (prednisone $60 \mathrm{mg} / \mathrm{m}^{2} /$ day) was started. Abdominal ultrasound showed discrete ascitic effusion. Intravenous albumin $(1 \mathrm{~g} / \mathrm{kg})$ infusion was administrated during the first day of hospitalization. For persistence of edema and oliguria, since the third day of hospitalization, therapy with furosemide was also started. Within the first 10 days after starting steroid therapy, the patient presented progressive increase

Alessia Morreale

alessiamorreale1989@gmail.com

1 Pediatric Unit, Carlo Poma Hospital, Mantua, Italy in diuresis, normalization of blood pressure values, weight loss, and increased serum calcium and albumin levels. Therapy with furosemide was gradually tapered and then discontinued. SARS-CoV-2 molecular test resulted negative 10 days after the first positive one. The patient was discharged within the first 14 days from onset of nephrotic syndrome with a weight of $13.6 \mathrm{~kg}$. Control blood tests after 20 days since onset of disease showed a complete normalization of IgG, fibrinogen, and antithrombin III values. Therapy with oral steroid was gradually tapered with disease remission.

Interestingly, recent case reports have described a temporal relation between COVID-19 mRNA vaccines and onset of nephrotic syndrome in adults, in particular with the development of minimal change disease in kidney biopsies, responsive to steroids $[2,3]$.

Further studies are needed to understand the pathogenesis of kidney involvement in COVID-19.

\section{Declarations}

Conflict of interest The authors declare no competing interests.

\section{References}

1. Alvarado A, Franceschi G, Resplandor E, Sumba J, Orta N (2021) COVID-19 associated with onset nephrotic syndrome in a pediatric patient: coincidence or related conditions? Pediatr Nephrol 36:205-207. https://doi.org/10.1007/s00467-020-04724-y

2. Holzworth A, Couchot P (2021) Minimal change disease following the Moderna mRNA-1273 SARS-CoV-2 vaccine. Kidney Int 100:463-464. https://doi.org/10.1016/j.kint.2021.05.007

3. Lebedev L, Sapojnikov M, Wechsler A, Varadi-Levi R, Zamir D, Tobar A, Levin-Iaina N, Fytlovich S, Yagil Y (2021) Minimal change disease following the Pfizer-BioNTech COVID-19 vaccine. Am J Kidney Dis 78:142-145. https://doi.org/10.1053/j.ajkd. 2021.03.010

Publisher's note Springer Nature remains neutral with regard to jurisdictional claims in published maps and institutional affiliations. 